TUM-T31-73/94

hep-ph/9408385n

\title{
Radiative Corrections to Nonleptonic Inclusive $B$ Decays and the Semileptonic Branching Ratio of B Mesons"
}

\author{
Patricia Ball \\ Physik-Department, TU München, D-85747 Garching, Germany
}

August 29, 1994

\begin{abstract}
:
We calculate the radiative corrections to the nonleptonic inclusive $\mathrm{B}$ decay mode $b \rightarrow c u d$ taking into account the charm quark mass. The corrections increase the decay rate by (4-8)\%, depending on the renormalization point. Using these results, we obtain an improved theoretical prediction for the semileptonic branching ratio of B mesons. This talk relies on work done in collaboration with E. Bagan, V.M. Braun and P. Gosdzinsky.
\end{abstract}

\section{Introduction}

Owing to the newly developed tool of an expansion in the inverse heavy quark mass [1], the theoretical description of weak inclusive decays of heavy mesons now rests on a more solid ground than ever. Since in such decays the energy release is large compared to the masses of the final state particles, the process takes place essentially at small distances and in leading order in the heavy quark expansion (HQE) is described by the underlying quark decay process. Hadronic corrections only enter at second order in the HQE and are of natural size $\sim 1 \mathrm{GeV}^{2} / m_{b}^{2} \sim 5 \%$ for B decays. Thus the accuracy of theoretical predictions of hadronic quantities like, say, the semileptonic branching ratio is not so much limited by the necessarily incomplete knowledge of (non-perturbative) hadronic matrix elements, but rather controlled by our knowledge of perturbative corrections to the free quark decay.

Full corrections to $\mathcal{O}\left(\alpha_{s}\right)$ are known for the semileptonic decay $b \rightarrow c e \nu$ [2], and for $b \rightarrow$ cud in the limit of massless final state quarks [3]. Although it is known that the exchange of gluons between quarks of unequal masses can yield big effects (cf. the extreme case of an infinitely heavy heavy quark investigated in [4]), in existing analyses of the semileptonic branching ratio of $\mathrm{B}$ mesons [5], $m_{c}$ was put zero in the radiative corrections to the nonleptonic width. In order to improve the existing predictions, we thus felt motivated to calculate the radiative corrections to $b \rightarrow c u d$ with full account for the c quark mass [6].

\footnotetext{
${ }^{*}$ Invited talk given at QCD 94, Montpellier, France, 7-13 July 1994; to appear in the Proceedings
} 


\section{Method of Calculation}

Without going into too much details, I present a short outline of the calculation done in [6]. Our starting point was to express the decay rate as imaginary part of the relevant forwardscattering amplitude. We used $\overline{\mathrm{MS}}$ subtraction and regularized occurring ultraviolet divergencies with dimensional regularization with anticommuting $\gamma_{5}$, often referred to as naïve dimensional regularization (NDR). NDR is applicable if one uses Fierz-transformations to relate diagrams with closed fermion loops, which are ambiguous in NDR, to such diagrams that are well-defined in NDR. As shown in [7], Fierz-transformations are valid diagram by diagram only with the proper choice of the so-called evanescent operators. We have verified that in the limit $m_{c} \rightarrow 0$ our procedure yields the same results as obtained in other schemes [3].

For the calculation of the imaginary parts of the forward-scattering amplitudes, we used a rather conservative technique, namely applied Cutkosky rules and regularized intermediate infra-red singularities by small quark and gluon masses which allows phase-space integration to be done in four dimensions.

\section{Results}

Since the complete formulas for $\Gamma(b \rightarrow c u d)$ are rather involved, I present results only in form of plots. Fig. 1 shows the effect of the non-vanishing c quark mass on the quark decay rate

$$
\Gamma(b \rightarrow c u d)=3 \Gamma_{0} \eta(\mu) J\left(m_{c} / m_{b}, \mu\right) .
$$

Here $\Gamma_{0}$ is the semileptonic tree-level decay rate, the factor 3 accounts for the colour enhancement in nonleptonic decays, $\mu$ is the renormalization scale, $\eta(\mu)$ contains the leading order QCD corrections, $\eta(4.8 \mathrm{GeV})=1.10$, and $J\left(m_{c} / m_{b}, \mu\right)$ gives the next-to-leading order corrections. In Fig. 1 the quantity $J\left(m_{c} / m_{b}, \mu\right) / J(0, \mu)$ is plotted as function of $m_{c} / m_{b}$ for three different values of the renormalization scale $\mu$. The grey bar denotes a conservative range of "physical" quark masses. Finite c quark mass effects thus constitute a $(4-8) \%$ increase of the decay rate $\Gamma(b \rightarrow c u d)$ with respect to the massless case.

Turning now to hadronic corrections, the HQE of $\Gamma(B \rightarrow X e \nu)$ involves to order $1 / m_{b}^{2}$ two hadronic matrix elements:

$$
\begin{aligned}
& 2 m_{B} \lambda_{1}=\left\langle B\left|\bar{b}_{v}(i D)^{2} b_{v}\right| B\right\rangle, \\
& 6 m_{B} \lambda_{2}=\left\langle B\left|\bar{b}_{v} \frac{g}{2} \sigma_{\mu \nu} F^{\mu \nu} b_{v}\right| B\right\rangle
\end{aligned}
$$

where $b_{v}$ is defined as $b_{v}=e^{i m_{b} v x} b(x), b(x)$ being the $b$ quark field in full QCD, $v_{\mu}$ is the four-velocity of the B meson, $m_{B}$ its mass and $F^{\mu \nu}$ the gluonic field-strength tensor.

Whereas $\lambda_{2}$ is directly related to the observable spectrum of beautiful mesons,

$$
\lambda_{2} \approx \frac{1}{4}\left(m_{B^{*}}^{2}-m_{B}^{2}\right)=0.12 \mathrm{GeV}^{2}
$$




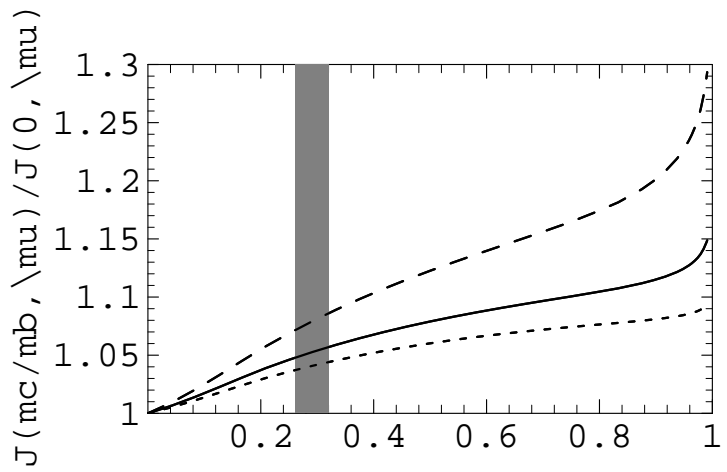

Figure 1: The next-to-leading order corrections $1\left(m_{n} n_{c} / m_{b}, \mu\right)$ to $\Gamma(b \rightarrow c u d)$ as function of $m_{c} / m_{b}$, normalized to one at $m_{c}=0$, for three different renormalization scales: solid line: $\mu=m_{b}$, long dashes: $\mu=m_{b} / 2$, short dashes: $\mu=2 m_{b}$.

the quantity $\lambda_{1}$ is difficult to measure, cf. [8]. Physically, $-\lambda_{1} /\left(2 m_{b}\right)$ is just the average kinetic energy of the $\mathrm{b}$ quark inside the meson. In the present analysis I conform to the value $\lambda_{1}=-(0.6 \pm 0.1) \mathrm{GeV}^{2}$ obtained from QCD sum rules [9]. For a discussion of the present status of $\lambda_{1}$, I refer to [10].

The semileptonic branching ratio is defined by

$$
B(B \rightarrow X e \nu)=\frac{\Gamma(B \rightarrow X e \nu)}{\Gamma_{t o t}}
$$

with

$$
\Gamma_{t o t}=\sum_{\ell=e, \mu, \tau} \Gamma\left(B \rightarrow X \ell \nu_{\ell}\right)+\Gamma\left(B \rightarrow X_{c}\right)+\Gamma\left(B \rightarrow X_{c \bar{c}}\right) .
$$

The explicit formulas for the decay rates can be found in [1]. In evaluating (田), it is crucial to minimize the number of independent parameters. To this purpose, we take advantage of the fact that the difference between heavy quark masses is fixed in the framework of HQE:

$$
m_{b}-m_{c}=m_{B}-m_{D}+\frac{\lambda_{1}+3 \lambda_{2}}{2}\left(\frac{1}{m_{b}}-\frac{1}{m_{c}}\right)+\mathcal{O}\left(\frac{1}{m^{2}}\right) .
$$

The only quantity remaining to be fixed is then $m_{b}$ or $m_{c}$. We prefer to take $m_{b}$ from spectroscopy and choose the most conservative range

$$
4.5 \mathrm{GeV} \leq m_{b} \leq 5.1 \mathrm{GeV},
$$

which is broad enough to cover all uncertainties arising from the renormalon ambiguity of the pole mass, cf. [11.

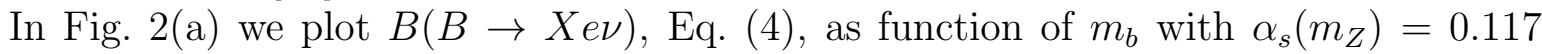
[12]. Here the widths are expressed in terms of pole quark masses. Nevertheless the pole masses are not the genuinely most suited ones for the analysis of weak decays, cf. [13].

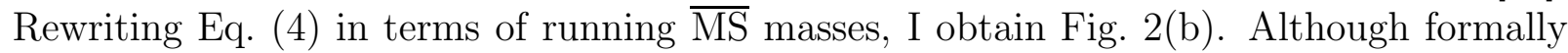
the difference to Fig. 2(a) is of higher order in $\alpha_{s}$, it is clearly visible and illustrates the 

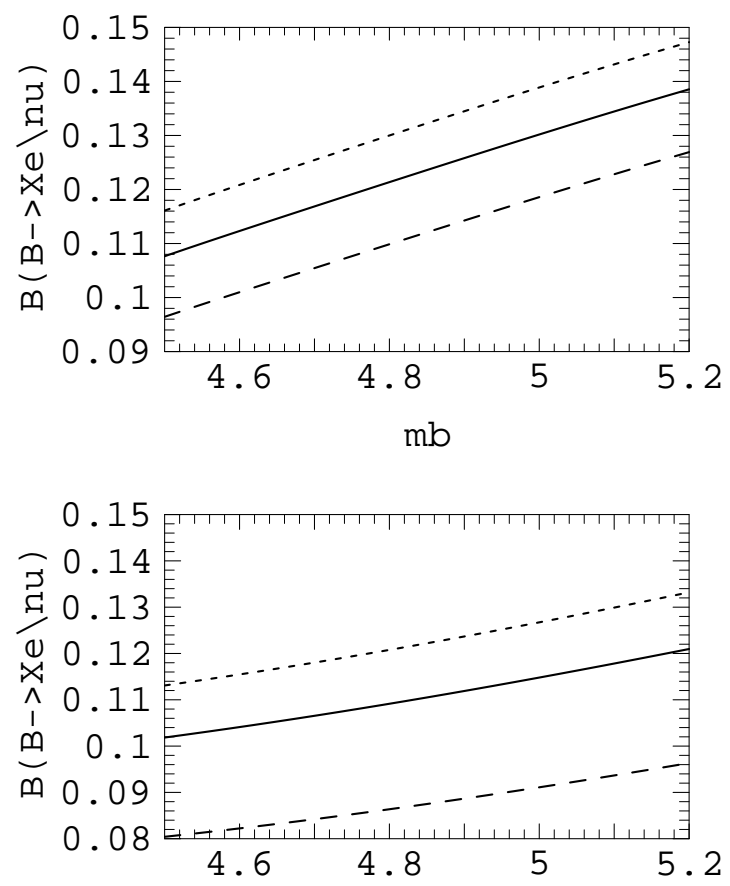

Figure 2: (a) $B(B \rightarrow X e \nu)$, Eq. (四), as functionmof the pole mass $m_{b}$ with $m_{b}-m_{c}$ fixed by Eq. (6). $m_{b}$ and $m_{c}$ are pole masses. The three lines have the same meaning as in Fig. 1. (b) The same as (a), but with running masses $m(\mu)$. Yet, as in (a), the abscissa is given in terms of the pole mass.

problem of scheme-dependence that makes its appearance in any finite order perturbative calculation.

From Fig. 2 we obtain

$$
B(B \rightarrow X e \nu)=(11.5 \pm 1.3 \pm 1.0) \%,
$$

where the first error combines uncertainties in $\alpha_{s}\left(m_{Z}\right), m_{b}$, the hadronic corrections, the renormalization scale and unknown radiative corrections to $b \rightarrow c c s$. The second error is a "guestimate" of the theoretical error due to scheme-dependence. Eq. (8) has to be compared with previous theoretical analyses, which consistently yielded $B(B \rightarrow X e \nu)>$ $12.5 \%$ [5], the experimental world average $B(B \rightarrow X e \nu)=(10.43 \pm 0.24) \%$ [14] and the most recent CLEO measurement $B\left(B^{0} \rightarrow X e \nu\right)=(10.9 \pm 0.7 \pm 1.1) \%$, [15]. The combined effect of complete radiative corrections, new results on $\alpha_{s}\left(m_{Z}\right)$ [12] and the consideration of different definitions of the quark mass thus lowers the theoretical branching ratio, which now agrees with the experimental one within the errors. A more detailed analysis is in preparation [16].

\section{References}


[1] I. Bigi, N. Uraltsev, A. Vainshtein, Phys. Lett. B 293 (1992) 430; Erratum ibid. 297 (1993) 477;

I. Bigi et al., Phys. Rev. Lett. 71 (1993) 496;

A. Manohar, M.B. Wise Phys. Rev. D 49 (1994) 1310;

B. Blok et al., Phys. Rev. D 49 (1994) 3356;

T. Mannel, Nucl. Phys. B413 (1994) 396

[2] N.Cabibbo, L.Maiani, Phys.Lett. B 79 (1978) 109;

Y. Nir, Phys. Lett. B 221 (1989) 184

[3] G. Altarelli et al., Nucl. Phys. B187 (1981) 461;

G. Buchalla, Nucl. Phys. B391 (1993) 501

[4] D.J. Broadhurst, A.G. Grozin, Phys. Lett. B 274 (1992) 421;

E. Bagan et al., Phys. Lett. B 278 (1992) 457;

M. Neubert, Phys. Rev. D 45, 2451 (1992)

[5] G. Altarelli, S. Petrarca, Phys. Lett. B 261 (1991) 303;

I. Bigi et al., Phys. Lett. B 323 (1994) 408

[6] E. Bagan et al., TU München Preprint TUM-T31-67/94 (hep-ph/9408306)

[7] A. Buras, P. Weisz, Nucl. Phys. B333 (1990) 66;

M. Jamin, A. Pich, Preprint CERN-TH.7151/94 (1994) (hep-ph/9402363);

S. Herrlich, U. Nierste, TU München Preprint TUM-T31-66/94 (in preparation)

[8] I. Bigi et al., Minneapolis Preprint TPI-MINN-94/25-T (hep-ph/9407296)

[9] P. Ball, V.M. Braun, Phys. Rev. D 49 (1994) 2472

[10] M. Neubert, these proceedings.

[11] I. Bigi et al., Phys. Rev. D 50 (1994) 2234;

M. Beneke, V.M. Braun, MPI München Preprint MPI-PhT/94-9 (hep-ph/9402364);

M. Beneke, V.M. Braun, V.I. Zakharov, MPI München Preprint MPI-PhT/94-18 (hep-ph/9405304)

[12] S. Bethke, these proceedings.

[13] P. Ball, U. Nierste, TU München Preprint TUM-T31-56/94/R (hep-ph/9403407) (to appear in Phys. Rev. D)

[14] M. Aguilar-Benitez et al. (Particle Data Group), Phys. Rev. D 50 (1994) 1173

[15] M.Athanas et al. (CLEO-coll.), Cornell Preprint CLNS 94-1286 (hep-ex/9406004)

[16] E. Bagan et al., TU München Preprint TUM-T31-68/94 (in preparation) 